\title{
Hochempfindliche Dünnschicht-Dehnungsmessstreifen auf technischen Bauteilen
}

Ralf Bandorf, Fraunhofer-Institut für Schicht- und Oberflächentechnik IST, Bienroder Weg 54 E, 38108 Braunschweig, Tel.-Nr. +49 531 2155-602, ralf.bandorf@ist.fraunhofer.de

Holger Gerdes, Fraunhofer-Institut für Schicht- und Oberflächentechnik IST, Bienroder Weg 54 E, 38108 Braunschweig, Tel.-Nr. +49 531 2155-576, holger.gerdes@ist.fraunhofer.de

Ulrike Heckmann, Fraunhofer-Institut für Schicht- und Oberflächentechnik IST, Bienroder Weg 54 E, 38108 Braunschweig, Tel.-Nr. +49 531 2155-581, ulrike.heckmann@ist.fraunhofer.de

Mirjana Petersen, Technische Universität Braunschweig, Institut für Oberflächentechnik, Bienroder Weg 54 E, 38108 Braunschweig, Tel.-Nr. +49 531 2155-684, mirjana.petersen@tu-braunschweig.de

Günter Bräuer, Fraunhofer-Institut IST und Technische Universität Braunschweig IOT, Bienroder Weg 54 E, 38108 Braunschweig, Tel.-Nr. +49 531 2155-501, guenter.braeuer@ist.fraunhofer.de

\section{Einleitung}

Gesputterte Dünnschicht-Dehnungsmessstreifen (DMS) besitzen eine Vielzahl von Vorteilen gegenüber konventionellen Folien-DMS. Sie können z. B. mit einem sehr hohen Automatisierungsgrad und hoher Positionsgenauigkeit aufgebracht werden. Zudem befindet sich zwischen Dünnschichtsensor und Bauteil nur eine wenige Mikrometer dicke keramische Isolationsschicht, anstatt einer aufgeklebten elastischen Trägerfolie, woraus eine erhöhte Messgenauigkeit resultiert. Aus den genannten Gründen werden gesputterte Dehnungsmessstreifen bereits heute in kommerziellen Drucksensoren in einer Vielzahl von Produkten zur Messung von statischen oder dynamischen Drücken eingesetzt.

Allerdings ist die maximale Empfindlichkeit der Sensoren, neben der eingesetzten Elektronik, durch die verfügbaren dehnungsempfindlichen Materialienbegrenzt. Die üblicherweise eingesetzten Legierungen, wie z. B. Nickel-Chrom, besitzen eine metalltypische Dehnungsempfindlichkeit mit einem k-Faktor von ca. 2. Sie werden insbesondere aufgrund ihres weitgehend temperaturunabhängigen Widerstands (mit einem Temperaturkoeffizient des elektrischen Widerstands $T K_{R}$ nahe $0 \mathrm{ppm} / \mathrm{K}$ ) eingesetzt, wodurch eine temperaturkompensierte Dehnungsmessung ermöglicht wird.

Neuartige piezoresistive Schichten erlauben eine deutliche Steigerung der Dehnungsempfindlichkeit im Vergleich zu konventionellen Materialen. Die piezoresistiven Materialien für die Sensorschicht bestehen idealerweise aus einer halbleitenden Matrix mit eingelagerten metallischen Nanopartikeln. Interessante Nanokomposit-Schichten sind z. B. nickelhaltige diamantähnliche Kohlenwasserstoffschichten (Ni-DLC). Der elektrischeWiderstand der DLC-Matrix hat einen negativen Temperaturkoeffizienten, die metallischen Partikel dagegen einen positiven Temperaturkoeffizienten. Das unterschiedliche Temperaturverhalten führt bei geeignetem Materialverhältnis zu einem temperaturunabhängigen elektrischen Widerstand. Darüber hinaus ist der Widerstand der Schichten abhängig vom Abstand der elektrisch leitfähigen Partikel untereinander und ändert sich somit deutlich stärker unter Dehnung als bei reinen Metallschichten.Mit NiDLC, welches auch unter weiteren Bezeichnungen, wieNi:a-C:H, a-C:H:Ni oder NanoNi@C, bekannt ist, können k-Faktoren mit Werten um die 20 bei gleichzeitig sehr niedrigen Temperaturkoeffizienten des elektrischen Widerstands $\left(\mathrm{TK}_{\mathrm{R}}< \pm 100 \mathrm{ppm} / \mathrm{K}\right)$ [Ban09, Pet11, Kop9, Kop10] erzielt werden.

Die sensorische Nutzung des piezoresistiven Verhaltens von metallhaltigen und reinen DLC-Schichten ist seit längerem bekannt und geschützt [Lue99]. Reine DLC-Schichten weisen mit k-Faktoren bis über 1000 noch weit höhere Dehnungsempfindlichkeiten auf als metallhaltige DLC-Schichten [Tib06, Bie06, Pet12]. In Abhängigkeit von den Beschichtungsparametern sind die DLC-Schichten sowohl als Drucksensoren im direkten Kraftschluss (Diaforce ${ }^{\circledR}$ ) sowie auch als Dehnungsmessstreifen einsetzbar. Auf Grund der hohen Temperaturabhängigkeit ist jedoch eine hochempfindliche Temperaturkompensation zu berücksichtigen. Einfacher ist die mikrotechnologische Anwendung von reinen DLC-Sensoren, wenn durch entsprechende Randbedingungen eine weitgehende Temperaturhomogenität im Sensorbereich gewährleistet wird.

Eine wichtige Voraussetzung für funktionsfähige Dünnschicht-DMS auf metallischen Bauteilen ist eine zuverlässige Isolationsschicht zwischen Bauteil und derSensorschicht. Hierfür stehen verschiedene Verfahren und Materialkombinationen zur Verfügung. Etabliert haben sich unter anderem $\mathrm{Al}_{2} \mathrm{O}_{3}-\mathrm{und}$ $\mathrm{SiO}_{2}$-Schichten [Fru05]. Während $\mathrm{SiO}_{2}$-Schichten, die mittels thermischer CVD-Verfahren abgeschieden 
werden, Prozesstemperaturen von über $500{ }^{\circ} \mathrm{C}$ benötigen [Mar91], bietet das HF-Sputtern von $\mathrm{Al}_{2} \mathrm{O}_{3}$ vom keramischen Target zuverlässige Isolation bei Temperaturen unterhalb von $300{ }^{\circ} \mathrm{C}$. Noch weiter reduziert wird die Prozesstemperatur bei der plasmaunterstützten CVD-Abscheidung (PACVD) von $\mathrm{SiO}_{2}$.

Die Strukturierung der Leiterbahnen, auch auf komplexen Oberflächen, sowie das Trimmen der Widerstände ist mittels moderner Lasertechnik möglich [Due11]. Alternativ kann die Strukturierung auch mittels klassischen Verfahren aus der Mikrotechnologie (photolithographisch und nasschemisch) oder mit Hilfe von Plasmaätzen erfolgen.

\section{Herstellung der Dehnungsmesstreifen auf technischen Bauteilen}

Zur Herstellung der Dehnungsmesstreifen auf dreidimensionalen technischen Bauteilen sind mindestens vier aufeinanderfolgende Prozessschritte erforderlich:

1. Bauteilvorbehandlung

2. Isolationsschichtabscheidung im Fall eines elektrisch leitfähigen Bauteils

3. Sensorschichtabscheidung

4. Strukturierung

Hinzu kommen die Qualitätskontrolle und das Trimmen des Widerstands der Dehnungsmesstreifen. Häufig werden die Kontaktbereiche vergoldet, um einen sicheren Löt- bzw. Bondprozess für die Anschlüsse zu ermöglichen. Je nach Anwendungsbereich ist auch eine Versiegelung des Sensors erforderlich. In Abbildung 1ist der prinzipielle Aufbau eines Dünnschicht-Dehnungsmessstreifens auf einem technischen Bauteil dargestellt. Abbildung 2 zeigt zwei verschiedene Demonstratoren.

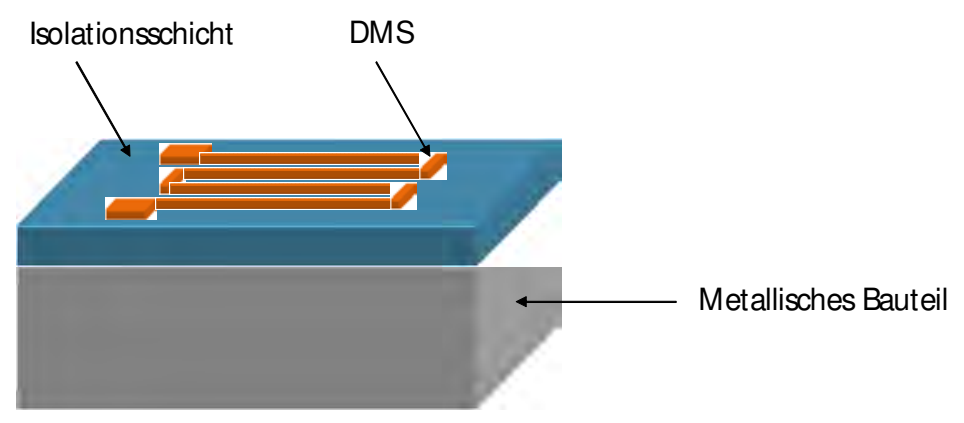

Abbildung 1: Schichtaufbau eines direktapplizierten Dünnschicht-Dehnungsmessstreifen auf einem metallischen Bauteil.
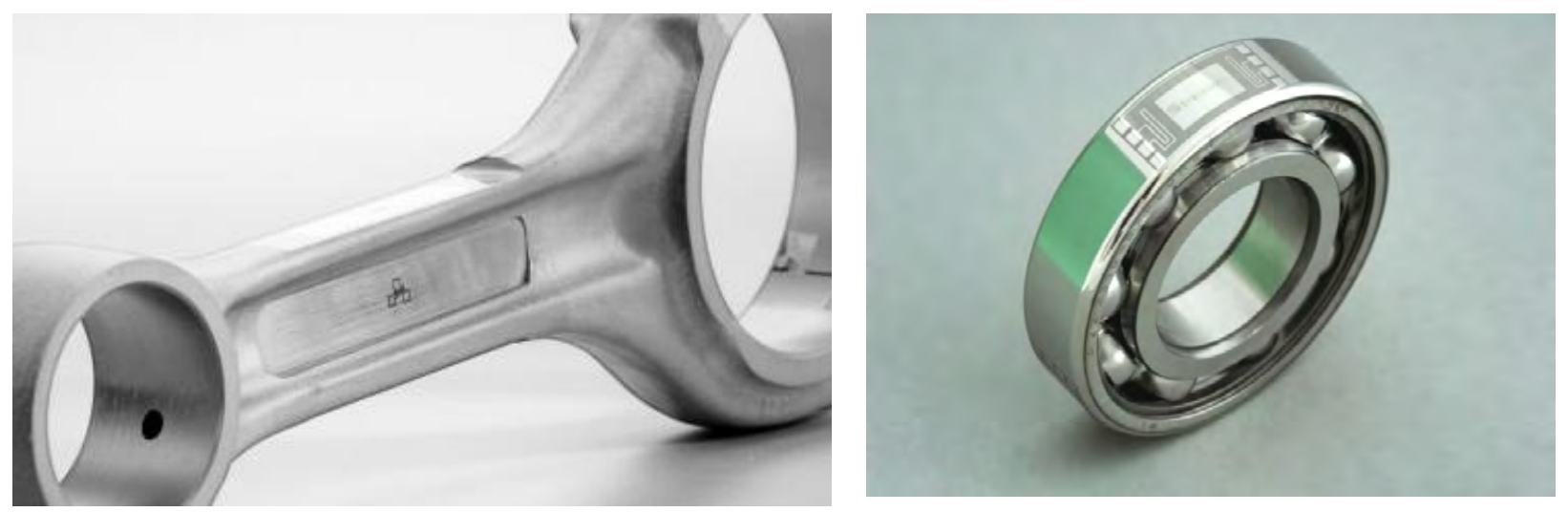

Abbildung 2: Beispiele von gesputterten Dünnschicht-Dehnungsmessstreifen auf technischen Bauteilen. Links: DMS auf Basis einer NiCr-Legierung auf einem LKW-Pleuel, rechts: vergleichbare DMS mit großen Strukturen zur Demonstration auf einem Lagerring. 


\subsection{Bauteilvorbehandlung}

Für eine zuverlässige Funktion des Sensorsystems wird der Dünnschichtsensor auf eine möglichst glatte Oberfläche aufgebracht. Hierfür müssen die metallischen Bauteilevor der Beschichtung gegebenenfalls nachpoliert werden. Um eine ausreichende Barrierewirkung der Isolationsschicht zu gewährleisten, ist es notwendig, dass die Bauteiloberfläche eine geringe Rauheit (mit niedrigen $R_{z}-$ Werten im Bereich der Schichtdicke der Sensorschicht) aufweist.Eine geringe Oberflächenrauheit ist bei DünnschichtDehnungsmesstreifen zudem auch Voraussetzung für eine reproduzierbare Dehnungsmessung und somit für eine hohe Messgenauigkeit, da hierdurch Störungen aufgrund topografischer Effekte vermieden werden.

Unmittelbar vor der Beschichtung erfolgt eine Reinigung in wässriger Lösung, da nur auf sauberen Oberflächen die dauerhafte Funktion der isolierenden und elektrischen Schichten gewährleistet werden kann[Mat10]. Die wässrige Reinigung deckt einbreites Reinigungsspektrum von derGrobreinigung bis zur Feinstreinigung ab, dabei erfüllt sie eineReihe komplexer Anforderungen:

- Intensive, aber schonende Reinigungverschiedener Werkstoffe, wieMetall, Keramik, Glas oderKunststoff.

- Vollständige Entfernung diverserVerunreinigungen, z. B. Öle, Kühlschmierstoffe,Späne, Rost, Salzeoder Schleifreste.

- Aktivierung oder Passivierung vonWerkstoffoberflächen und Vorbereitungfür die nachfolgende Bearbeitung.

- Korrosionsschutz während undnach der wässrigen Reinigung.

Im Anschluss an die wässrige Reinigung erfolgt die aktive Trocknung in einem kombinierten Umluft- und Vakuumtrocknungs-System (WETex ${ }^{\circledR}$ ), das auch mit Schutzgas betrieben werden kann und somit auch für korrosionsempfindliche Werkstoffe geeignet ist.

\subsection{Beschichtungstechnik}

Bei den verwendeten Verfahren handelt es sich um Vakuum-Beschichtungsverfahren mittels Plasmatechnologie im Hochvakuum. In der Prozesskammer wird zwischen Kathode und Anode (meist Kammerwand) mit Hilfe eines Prozessgases und einer hohen elektrischen Spannung ein Plasma gezündet. Das Plasma trägt je nach Prozess dazu bei die Kathode zu zerstäuben (Sputtern), damit die herausgeschlagenen Atome sich auf dem Substrat niederschlagen können. Alternativ oder ergänzend kann ein Reaktivgas im Plasma angeregt werden und zur Schichtbildung beitragen [Cha80].

Zur Beschichtung der Bauteile stehen am Fraunhofer IST verschiedene HochvakuumBeschichtungsanlagen zur Dünnschicht-Abscheidung zur Verfügung. Für die hier vorgestellten Prozesse werden drei verschiedene Anlagentypen verwendet. Häufig eingesetzt wird ein Box-Coater(BAS 450, Balzers) mit viervertikal angeordneten Sputtertarget-Stationen der Größe $250 \times 125 \mathrm{~mm}^{2}$. Indieser Prozesskammer können sowohl flache als auch dreidimensionale Bauteile $\left(\varnothing_{\text {MAX }}: 300\right.$ mm, H: ca. $250 \mathrm{~mm}$ ) mit Hilfe von Substratrotation und Substrat-Bias-Spannung gleichmäßig beschichtet werden.Neben dem Box-Coater, der für jeden Substratwechsel belüftet und wieder evakuiert werden muss, stehen auch eine In-Line-Anlage (SV 470, FHR Anlagenbau)und eine Kurztakt-Anlage (IC 300, Impact Coatings)zur Verfügung. Diese beidenAnlagen sind mit einer Schleuse ausgestattet und ermöglichen die Beschichtung großer Stückzahlen von Substraten ohne Belüftung der Prozesskammer. Die In-Line-Anlage ist eine Entwicklungsanlage mit verschiedensten vertikal angeordneten SputterKathoden bis zu einer Länge von470 mm. Sie ist in der Lage bewegte und rotierende Substrate bis zu einer Fläche vonca. 300x 300 mm², bzw. einem Durchmesser von120 mm und einer Höhe von 300 mmzu beschichten. Die Kurztaktanlage, die sowohl für eine Beschichtung von oben nach unten als auch von unten nach oben geeignet ist, ist dagegen für kleinere Substrate bis maximal ca. $100 \times 200 \times 40 \mathrm{~mm}^{3}$ ausgelegt. Sie ist vom Grundkonzept her eine industrielle Produktionsanlage und ermöglicht durch kurze Abpumpzeiten der Schleuse einen sehr hohen Durchsatz.

Allgemein müssen alle nutzbaren Vakuumbeschichtungsanlagen über entsprechende Sputter-Stationen und idealerweise über eine Vorbehandlungsstation zum Ätzen oder Beglimmendes Substrats verfügen. Voraussetzung istein Vakuumpumpstand, der in der Regel aus Vorpumpe und Turbomolekularpumpe besteht. Sinnvoll ist ein Basisdruck im Bereich unterhalb von $10^{-5} \mathrm{mbar}\left(10^{-3} \mathrm{~Pa}\right)$, der Arbeitsdruck liegt dagegen häufig zwischen 0,5 und $2 \mathrm{~Pa}$.Die Prozessgasströme werden mit Hilfe von 
Massendurchflussreglern gesteuert. Ein Prozesstransfer auf vorhandene Anlagen bei Industrieunternehmen ist generell möglich. Notwendig ist dabei eine spezifische Anpassung der einzelnen Prozessschritte durch Fachpersonal.

\subsection{Isolationsschichten}

Eine wesentliche Voraussetzung für eine ausreichende elektrische Barriere zwischen dem auf einem elektrischen Widerstand basierenden Dehnungsmessstreifen und dem metallischen Bauteil ist eine zuverlässige Isolationsschicht. Diese Isolationsschicht sollte einen ausreichend hohen elektrischen Widerstand ohne Löcher oder Risse aufweisen, damit im Betrieb ein elektrischer Kurzschluss zwischen Bauteil und Sensor vermieden werden kann.

\subsubsection{Partikelminimierung}

Vor der eigentlichen Abscheidung der Isolationsschicht ist es notwendig die Partikelanzahl auf dem Substrat zu minimieren. Hierdurch können kleine Löcher (sogenannte „pinholes“) vermieden werden, die durch Partikel auf dem Substrat entstehen. Die Partikel werden bei der Abscheidung der Isolationsschicht ebenfalls beschichtet und können anschließend zum lokalen Abplatzen der Schicht in diesen Bereichen führen [Glo11]. Hauptverursacher für Partikel auf dem Substrat sind unzureichende Reinigung und unsachgemäßes „Handling“ der Substrate, weiterhin lässt es sich nicht vermeiden, dass sich in der Beschichtungskammer Partikel bilden, die sich beim Evakuieren, Beschichten (insbesondere bei der Beschichtung von oben nach unten oder beim „Arcing“) und Belüften auf das Substrat gelangen können.Durch Schulung des Personals und eine Reinraumumgebung im Bereich der Anlagenbestückung kann ein Großteil der Partikel vermieden werden. Darüber hinaus kann mit Hilfe geeigneter Maßnahmen die Partikelbelastung in der Beschichtungskammer signifikant reduziert werden.

In Abbildung 3 ist eine Partikelzählung auf Si-Wafern mittels mikroskopischer Charakterisierung und angepasster Bildverarbeitungin Abhängigkeit des Anlagenzustands und der In-situSubstratvorbehandlung dargestellt. Gezählt wurden Partikel mit einemDurchmesser unterhalb von $3 \mu \mathrm{m}$.Größere Partikel traten nur vereinzelt auf. Bei der gewählten Auswertunghandelt es sich bei den angegebenen Partikelzahlen, aufgrund von nicht vermeidbaren Pixelfehlern, um eine „Abschätzung nach oben“. Im Box-Coater ist ein deutlicher Anstieg der Partikelanzahl bei Nutzung der rotierenden Substrathalterung zu beobachten, die Partikelanzahl lässt sich mit Hilfe einer kontrollierten, $d$. h. in diesem Fall „sanfteren“, Evakuierung und Belüftung der Kammer auf ca. 25 \% reduzieren, jedoch erübrigt sich das kontrollierte Be- und Entlüften der Prozesskammer bei Nutzung eines Plasma-Ätzprozesses vor der eigentlichen Beschichtung. Die Anzahl der Partikel pro $\mathrm{cm}^{2}$ bleibt dennoch im Bereich von ca. 500 . In der In-Line-Anlage wurde eine drastische Partikelminimierung durch die Reinigung der Schleusenkammer nachgewiesen. Durch das Einfahren in die Prozesskammer kommt es zu einer weiteren

Partikelbelastung. Mit Hilfe eines speziellen Ätzprozesseslässt die Anzahl der Partikel pro $\mathrm{cm}^{2}$ jedoch auf unter 100 reduzieren. Die niedrigere Partikelanzahlin der In-Line-Anlage beruht im Wesentlichen darauf, dass nicht die Prozesskammer, sondern nur die Schleuse belüftet wird. Um die geringe Partikelbelastung dauerhaft zu gewährleisten, ist eine regelmäßige Anlagenreinigung erforderlich. 


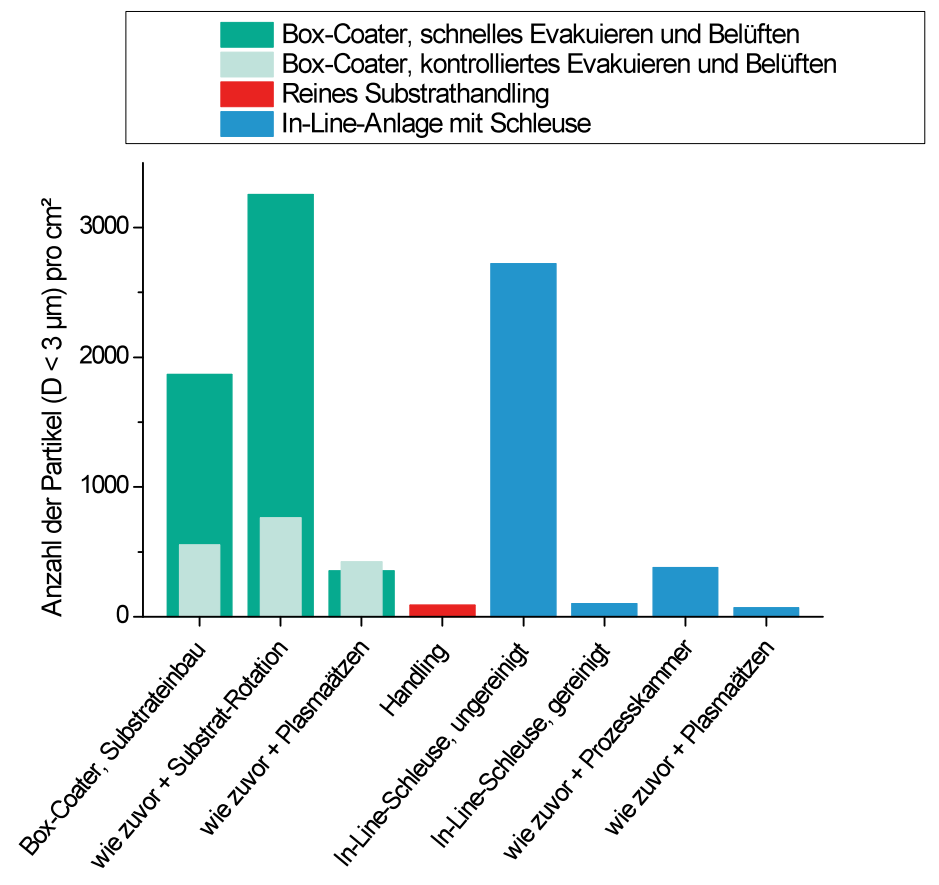

Abbildung 3: Partikelanzahl pro $\mathrm{cm}^{2}$ auf Testsubstraten nach verschiedenen Prozessschritten, die der Abscheidung der Isolationsschicht vorangehen. Optimal ist die Nutzung einer gereinigten Schleuse.

\subsubsection{Charakterisierung der Isolationswirkung}

Zur Charakterisierung der Isolationsschichten wird eine Durchschlagsfestigkeitsprüfungmit einem Hochspannungsmessgerät (ELABO TYP 91-1A)durchgeführt.Für die Prüfung werden die Isolationsschichten auf einem $2 \mathrm{~cm} \times 6 \mathrm{~cm}$ großen Edelstahlsubstrat großflächig metallisiert (z. B. mit Chrom/Gold). Die Metallschicht wird mit einer Prüfnadel kontaktiert. Zwischen Nadel und leitfähigem Substrat wird eine Prüfspannung angelegt und stufenweise auf $1 \mathrm{kV}$ erhöht, wobei bei einer Spannung von $0,05 \mathrm{kV}, 0,1 \mathrm{kV}$ und $0,5 \mathrm{kV}$ die jeweilige Spannung $10 \mathrm{~s}$ und bei einer Spannung von $1 \mathrm{kV}$ die Spannung $30 \mathrm{~s}$ gehalten wird. Die Messung wird beendet, sobald ein elektrischer Strom mit einer Stärke von $0,5 \mathrm{~mA}$ fließt, d. h. ein elektrischer Durchschlag vorliegt. Die Durchschlagsspannung $U_{\mathrm{D}}$ ist die Spannung, die erforderlich ist, um einen Strom von 0,5 mA fließen zu lassen.

\subsubsection{Herstellung und Eigenschaftenverschiedener Isolationsschichten}

\section{Aluminiumoxid $\left(\mathrm{Al}_{2} \mathrm{O}_{3}\right)$}

Reaktive Sputterprozesse zur Herstellung von Metalloxidschichten, wie z. B. $\mathrm{Al}_{2} \mathrm{O}_{3}$, sind seit langer Zeitbekanntund die Prozessregelung wird zunehmend verbessert [Kel96, Sch97, Bar02]. Nicht zuletzt durch die ermutigenden Ergebnisse aus der jüngsten Entwicklung im Bereich der Sputtertechnologie, dem Hochleistungsimpuls-Magnetronsputtern, kann davon ausgegangen werden, dass diese Art der Isolationsschichtherstellung sich zukünftig auch im industriellen Maßstab durchsetzten wird [Wal08, Aie11, Ver11]. Aktuell wird jedoch auf metallischen Bauteilen mit dem klassischen HF-Sputterprozess vom keramischen $\mathrm{Al}_{2} \mathrm{O}_{3}$-Target nach wie vor die zuverlässigste Isolationswirkungerzielt. Am Fraunhofer IST erfolgt die Beschichtung im bereits beschriebenen Box-Coater. Hierfür werden die gereinigten Proben auf einem rotierendem Substrathalter befestigt und anschließend wird die Prozesskammer evakuiert. Zur Substratvorbehandlung folgen einPlasmaätz-Prozess und die Abscheidung einer Titan-

Haftvermittlerschicht. Die Abscheidung der 4-5 $\mu$ m dicken $\mathrm{Al}_{2} \mathrm{O}_{3}$-Schicht erfolgt bei einem Arbeitsdruck von 0,5 Pa und einer HF-Leistung am Target von bis zu 2,5 kW.So kann bei geeigneter Prozessführung eine Durchschlagsspannung von über $0,5 \mathrm{kV}$ reproduzierbar erreicht werden. Nachteilig ist die geringe Abscheiderate (ca. $280 \mathrm{~nm} / \mathrm{h}$ ) und die daraus resultierende Prozessdauersowie die verhältnismäßig hohe Prozesstemperatur ( $\mathrm{T}_{\text {suB }}$ : ca. $210^{\circ} \mathrm{C}$ ). Zudem ist die Aufskalierungder HF-Sputterprozess auf große Industriekathoden sehr kostenintensiv. Hier werden zukünftig diereaktiven Sputterprozesse eine attraktive Alternative darstellen. 


\section{Siliziumdioxid $\left(\mathrm{SiO}_{2}\right)$}

Eine Isolationsschicht aus $\mathrm{SiO}_{2}$, die mit Hilfe eines PACVD-Prozesses hergestellt wird, bietet sich alternativ zum HF-gesputterten $\mathrm{Al}_{2} \mathrm{O}_{3}$ sowie zum $\mathrm{SiO}_{2}$ aus thermischen CVD-Prozessen an. $\mathrm{Am}$ Fraunhofer IST befindet sich dieser Prozess aktuell in der Erprobungsphase. Abbildung 4 zeigt $\mathrm{SiO}_{\mathrm{X}}$ Schichten nach einer Variation des Mischungsverhältnisses derProzessgase Sauerstoff $\left(\mathrm{O}_{2}\right)$ und Tetramethylsilan(TMS) auf Testsubstraten. Vorteilhaft ist insbesondere die niedrige Substrattemperatur. Die Isolationsschicht kann bei unter $150^{\circ} \mathrm{C}$ auch auf temperaturempfindliche Substrate aufgebracht werden.Darüber hinaus kann die Prozesszeit erheblich reduziert werden. Zurzeit können $5 \mu \mathrm{m}$ dicke $\mathrm{SiO}_{2}$-SchichtenmitAbscheideraten von ca. $20 \mathrm{~nm} / \mathrm{min}$ in gut 4 Stunden hergestellt werden. Bei der Schichtherstellung wird am Substrat eine Bias-Spannung im Bereich von -400 bis $-500 \mathrm{~V}$ angelegt. Die Prozessgase $\mathrm{O}_{2}$ undTMS werden mit einem Gasfluss im Bereich 100 bis $200 \mathrm{sccm}$ in die Prozesskammer des Box-Coaters eingelassen, der Prozessdruck liegt idealerweise im Bereich 0,5 bis $1 \mathrm{~Pa}$. Die mit den angegebenen Parametern hergestellten Isolationsschichten erreichen bereits teilweise die angestrebte Durchschlagsspannungen von über $0,5 \mathrm{kV}$, jedoch noch nicht mit der gleichen Zuverlässigkeit und Reproduzierbarkeit wie bei den zuvor beschriebenen $\mathrm{HF}-\mathrm{Al}_{2} \mathrm{O}_{3}$-Schichten.

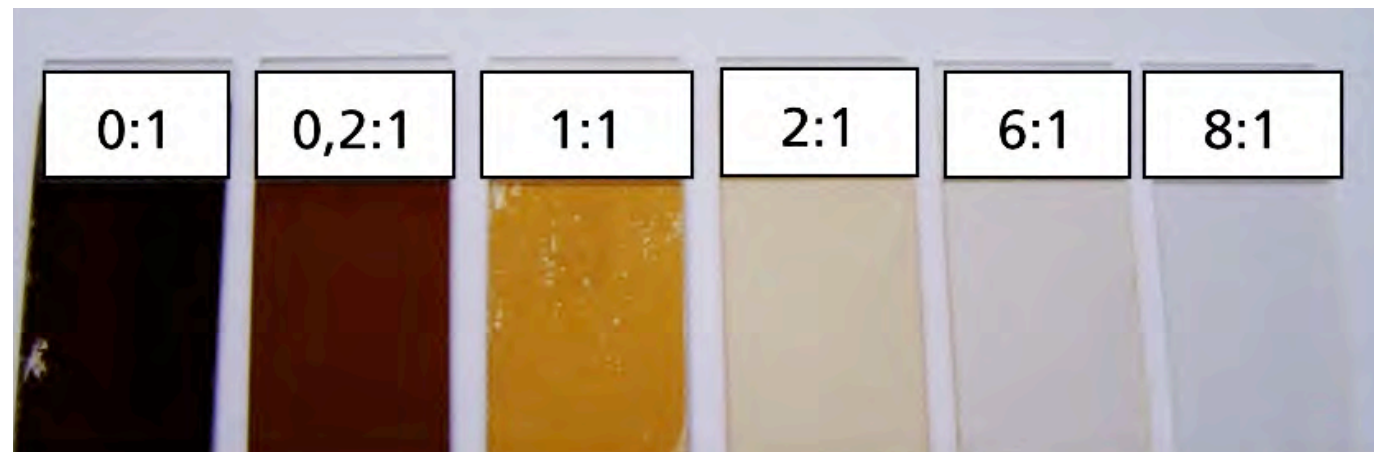

Abbildung 4: $\mathrm{SiO}_{x}$ auf Glassubstraten - Änderungen im Prozessgas-Mischungsverhältnis $\mathrm{O}_{2}:$ TMS führen zur stöchiometrischen Abscheidung und damit zu transparenten $\mathrm{SiO}_{2}$-Schichten.

\subsection{Sensorschichten für Dehnungsmessstreifen}

Sensorschichten für Dehnungsmessstreifen sind in erster Linie Widerstandsschichten, da die Dehnungsmessung auf der Widerstandsänderung einer Leiterbahn unter Dehnung oder Stauchung beruht. Der sogenannte k-Faktor ist als Proportionalitätsfaktor zwischen relativer Längenänderung bzw. Dehnung $(\Delta L / L)$ und relativer Widerstandsänderung $\left(\frac{\Delta R}{R}\right)$ das Maß für die Dehnungsempfindlichkeit des Materials:

$$
\frac{\Delta R}{R}=k \cdot \frac{\Delta L}{L}
$$

Der k-Faktor ist eine materialabhängige Größe. Wie von Parker und Krinsky [Par 63] gezeigt, besteht er für kleine Längenänderungen aus zwei Termen:

$k=(d R / R) /(d L / L)=(1+2 \sigma)+\left({ }^{d \rho} / \rho\right) /(d L / L)$

Der „geometrische“ Term $(1+2 \sigma)$ enthält die materialabhängige Poissionzahl $\sigma$, die bei den meisten Metallen im Bereich von 0,3 bis 0,45 liegt und maximal gleich 0,5 ist, so dass der geometrische Term des k-Faktors maximal den Wert von 2 annehmen kann. Der „physikalische“ Term $(d \rho / \rho) /(d L / L)$ ist bei den
meisten Metallen zu vernachlässigen, da sich deren spezifischer Widerstand $\rho$ unter Dehnung nicht 
verändert. Materialien mit einer hohen Dehnungsempfindlichkeit (k-Faktor > 2) müssen darum Materialien sein, deren spezifischer Widerstand eine dehnungsabhängige Größe ist. Dies trifft z. B. auf Halbleiter zu. Hohe k-Faktoren von Silizium und Germanium sind seit den 50er Jahren des vergangen Jahrhunderts bekannt [Smi54] und auch von diamantähnlichen Kohlenstoffen (DLC) wurden bereits hohe k-Faktoren veröffentlicht [Tib06].Da Halbleiter aber einen hohen negativen Temperaturkoeffizienten des elektrischen Widerstands $\left(\mathrm{TK}_{\mathrm{R}}<0\right)$ aufweisen, sind sie auch bei Verwendung von Brückenschaltungen meist nur mit hohem elektronischen Kompensationsaufwand als Dehnungssensoren einsetzbar.Abhilfe können heterogen zusammengesetzte Schichten bieten. Mit Nanokompositen, bestehend aus einer halbleitenden Matrix $\left(\mathrm{TK}_{R}<0\right)$ und metallischen Partikeln $\left(\mathrm{TK}_{R}>0\right)$, können bei geeigneter Zusammensetzung kFaktorengrößer 2 in Kombination mit einemTK $\mathrm{R}_{\mathrm{R}}$ nahe Nullerzielt werden.

Für die Herstellung von Dehnungsmessstreifen auf dreidimensionalen metallischen Werkstücken, die sich für industrielle Anwendungen eignen, ist ein $\mathrm{TK}_{\mathrm{R}}$ nahe Null neben der Stabilität die wichtigste Voraussetzung für eine kostengünstige, zuverlässige und reproduzierbare Signalmessung.

\subsubsection{Charakterisierungsmethoden}

Zur Charakterisierung der untersuchten Sensormaterialien stehen am Fraunhofer IST, neben der taktilen Schichtdickenmessung, der Vierspitzenmessung für den Schichtwiderstand und der ElektronenstrahlMikroanalyse (EPMA - zur chemischen Zusammensetzungsanalyse), verschiedene Messplätze zur Bestimmung des k-Faktors, des Temperaturkoeffizienten und der Widerstandsdrift bei Raumtemperatur und bei erhöhter Temperatur zur Verfügung. Zur Materialcharakterisierung werden Sensorschichten mit einer Dicke von 150 bis $1500 \mathrm{~nm}$ auf Substrate mit den Abmaßen: 2 × $6 \mathrm{~cm}^{2}$ abgeschieden. Als Substratmaterialen wird Aluminiumoxidkeramik (Dicke: 0,38 mm) und Glas (Dicke: 0,7 $\mathrm{mm}$ ) verwendet. Die Substrate werden während der Beschichtung mit einer Schattenmaske abgedeckt, die eine einfache Teststruktur ohne nachtäglichen Strukturierungsaufwand ermöglichen (sieheAbbildung 5). Teilweise wird die Querstruktur und der Kontaktbereich vergoldet.

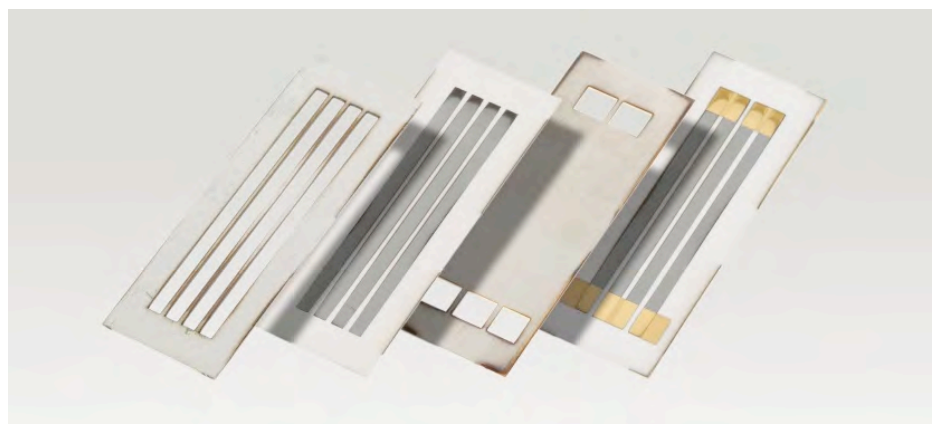

Abbildung 5: Herstellung einfacher Teststrukturen mit Hilfe von Schattenmasken.

\section{k-Faktor}

Die Bestimmung der Dehnungsempfindlichkeit erfolgt bei der Dehnung der Testsubstrate auf einen festen Radius von $750 \mathrm{~mm}$. Der elektrische Widerstand der Mäanderbahn wird im ungedehnten und gedehnten

Zustand gemessen. Die erzielte Dehnung $\varepsilon=\Delta L / L$ ist von der Dicke des verwendeten Substratmaterials abhängig(e = 0,3 \%o für die verwendeten Keramiksubstrate und e = 0,5\% für die verwendeten Glassubstrate). Die Messung und Auswertung erfolgt bei Raumtemperatur automatisiert und kann in einem klimatisierten Labor auch für Langzeitmessungen eingesetzt werden. Nach dem gleichen Prinzip erfolgt auch die Messung unter höheren Temperaturen. Hierfür kann eine Aufnahme aus keramischen Materialien in einem Hochtemperaturofen genutzt werden (sieheAbbildung 6). Aufnahme, Messdrähte und Ofen sind für Temperaturen bis $1200^{\circ} \mathrm{C}$ ausgelegt und auch eine k-Faktor-Bestimmung ist grundsätzlich bis zu dieser Temperatur möglich. 


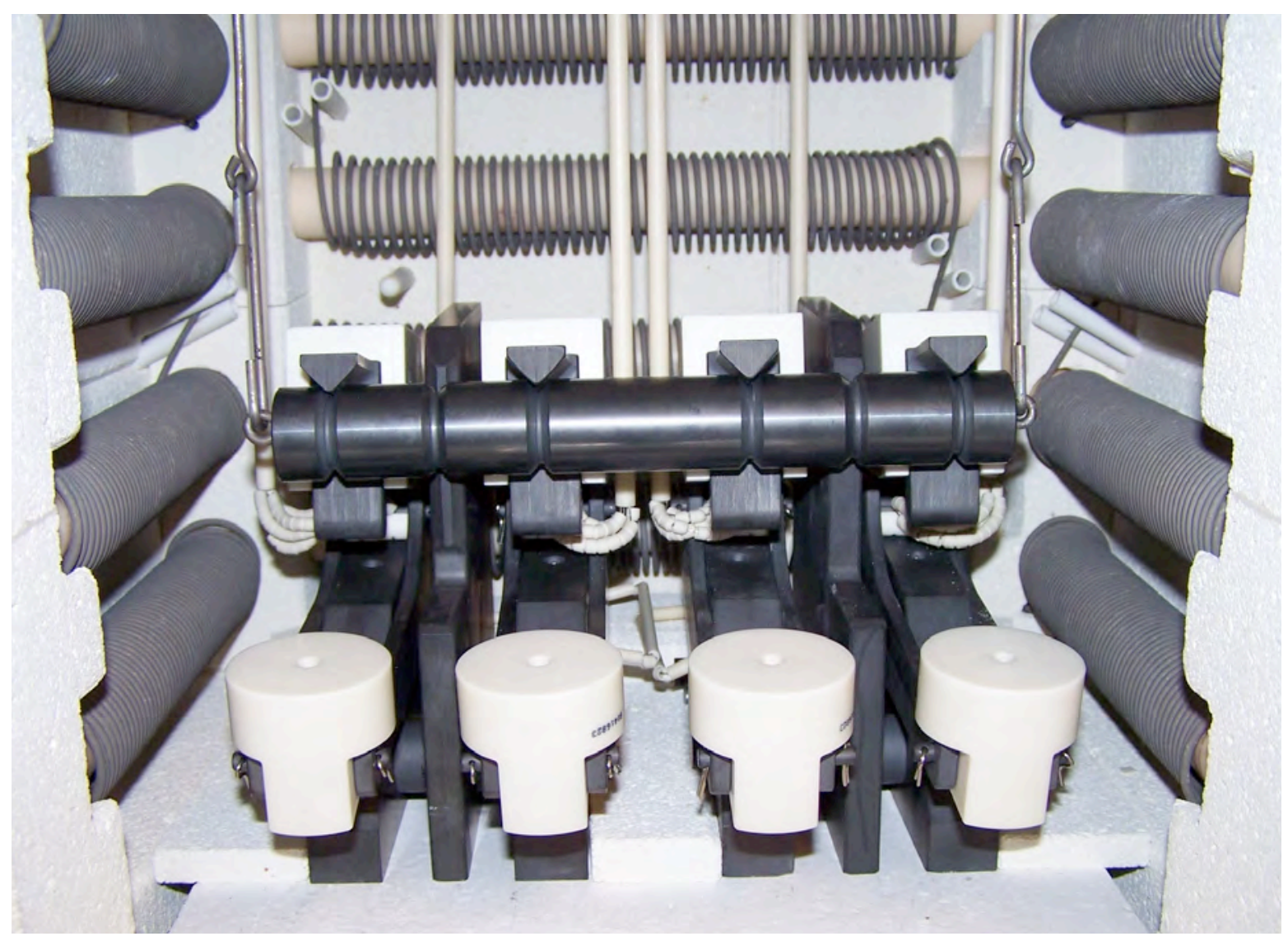

Abbildung 6: Blick in einen Hochtemperaturofen mit vier integrierten Messeinrichtungen zur automatisierten Bestimmung von k-Faktor und TCR.

\section{$T_{K_{R}-\text { Bestimmung }}$}

Für den Temperaturkoeffizient des elektrischen Widerstands wird das Testsubstrat in der Regel eine halbe Stunde bei einer Temperatur von $160{ }^{\circ} \mathrm{C}$ gehalten. Während des anschließenden Abkühlvorgangs wird die Temperaturabhängigkeit des Widerstands gemessen und nach dem bekannten Zusammenhang ausgewertet:

$T K_{R}\left(T_{R \theta f}\right)=\left.\frac{1}{R\left(T_{R \theta f}\right)} \cdot \frac{d R(T)}{d T}\right|_{T=T_{R e f}}$

Auch im Anschluss an eine Hochtemperaturbehandlung ist je nach $R(T)$-Verlauf die Bestimmung des $T_{K}$ möglich.

\section{Widerstandsdrift}

Aufgrund der guten Temperaturstabilität eignet sich der Hochtemperaturmessplatz ideal zur Prüfung des Langzeitverhaltens des elektrischen Widerstands der Sensorschichten. Ausgehend vom Anfangswert wird die relative Widerstandsänderung in $\mathrm{ppm} / \mathrm{h}$ in Abhängigkeit von der jeweiligen Umgebungstemperatur angegeben.

\subsubsection{Sensormaterialien und Herstellungsmethoden}

Konventionell werden temperaturkompensierte Legierungen auf der Basis von Nickel-Chrom-Legierungen $(\mathrm{NiCr})$ oder Konstantan als Material für Dehnungsmesstreifen verwendet. Für gesputterte DünnschichtDehnungsmessstreifen haben sich die sehr korrosionsbeständigen NiCr-Legierungen durchgesetzt.

Diese Legierungen lassen sich durch geeignete Zusammensetzung der Legierungsmaterialien,

Abscheidebedingungen und Temperschritte so auf das jeweilige Substrat abstimmen, dass der TK $\mathrm{T}_{\mathrm{R}}$ nur 
wenige ppm/K beträgt. Für hohe Dehnungsempfindlichkeiten besitzt aktuell Nickel-DLC das höchste Potenzial im Anwendungsbereich von Raumtemperatur bis ca. $150{ }^{\circ} \mathrm{C}$. Es wird mit dem zuvorbeschriebenen reaktiven Sputterprozesshergestellt. Zusätzlich zum HF-Sputter-Prozess vom Nickel-Target in Argon-Atmosphäre wird ein reaktives kohlenstoffhaltiges Prozessgas in die Prozesskammer eingelassen (z. B. $\mathrm{C}_{2} \mathrm{H}_{2}$ ). Bei geeigneter Wahl der Prozessparameter scheidet sich auf dem Substrat eine amorphe Kohlenwasserstoffschicht mit eingelagerten Nickelpartikeln und vielversprechenden sensorischen Eigenschaften ab.

\subsubsection{Parametervariation und Ergebnisse}

\section{Nickel-Chrom-Legierungen als DMS-Material}

Der Herstellungsprozess für NiCr-Dünnschicht-DMS ist ein klassischer DC- bzw. HF-Sputterprozess in Argon-Atmosphäre. Als Sputter-Kathode wird ein Komposit-Target mit einer Zusammensetzung von beispielsweise $80 \mathrm{Gew} .-\% \mathrm{Ni}$ und $20 \mathrm{Gew} .-\% \mathrm{Cr}$ oder $40 \mathrm{Gew} .-\% \mathrm{Ni}$ und $60 \mathrm{Gew} .-\% \mathrm{Cr}$ verwendet.Mit Hilfe einer Substratrotation können dreidimensionale Bauteile homogen beschichtet werden. Nach einem Temperschritt bei ca. $300^{\circ} \mathrm{C}$ werden die Dünnschichtwiderständekommerziell bei Temperaturen bis ca. $150{ }^{\circ} \mathrm{C}$ mit niedrigen $\mathrm{TK}_{\mathrm{R}}$-Werten, sehr geringer Drift und einem k-Faktor von ca. 2 eingesetzt.Verschiedene Hersteller verwenden niedrige Al- oder Si-Zulegierungen, wodurch die Dehnungsempfindlichkeit und jedoch nicht beeinflusst wird.

\section{Nickel-DLC als DMS-Material}

Ni-DLC wird zurzeit noch nicht kommerziell eingesetzt. Es existieren jedoch bereits Rezepte für reproduzierbare Prozesse, die bei einem Prozessdruck von 1-2 Pa und einer niedrigen Bias-Spannung am Substrat - ohne große Parameteroptimierungen und zusätzlicher Substratheizung - im statischen und dynamischen Beschichtungsprozess zu k-Faktoren $>10$ in Kombination mit einem

Temperaturkoeffizienten $<+/-100$ ppm/K führen. Diese Prozesse benötigen aktuell aber noch eine im HF-Mode betriebene Nickel-Kathode, welche industriell jedoch selten Verwendung findet. Abbildung 7 zeigt die in erster Näherung lineare Abhängigkeit des $\mathrm{TK}_{\mathrm{R}}$ vom Nickelgehalt in der Ni-DLC-Schicht. In Abbildung 8 ist der k-Faktor von Ni-DLC-Schichten dargestellt, die bei nicht optimierten Prozessparametern auf statischen und rotierenden Substraten abgeschieden wurden. Obwohl der kFaktor der statischen Schichten im Mittel höher ist als der k-Faktor der rotierenden Schichten, sind die Maximalwerte im k-Faktor bei gut 55 At.-\% Nickel mit Werten um die 10 jedoch vergleichbar. Abbildung 9zeigt das Potenzial der Ni-DLC-Schichten, mit k-Faktoren im Bereich von 17 bei optimiertem Prozessdruck und z. T. zusätzlicher Substratheizung.

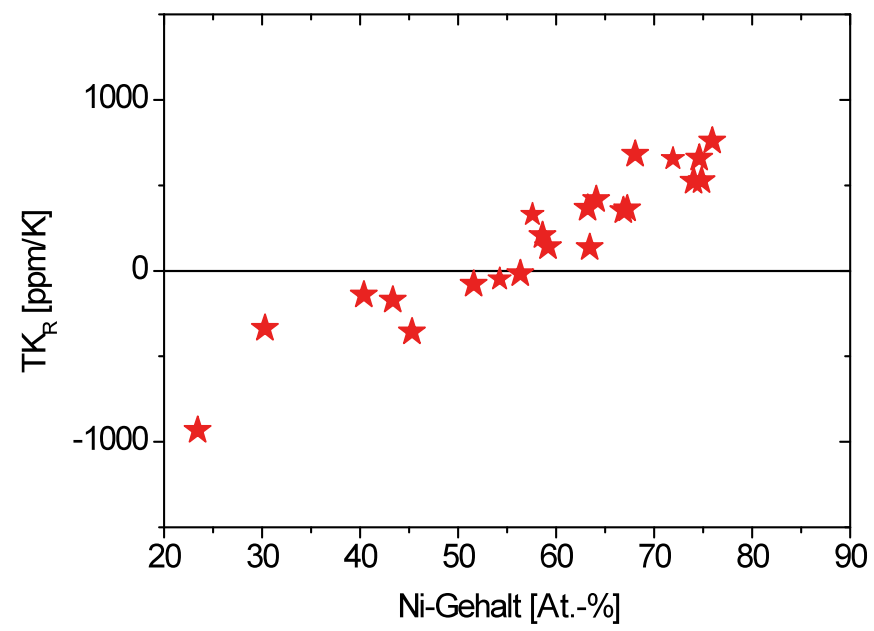

Abbildung 7: Der Temperaturkoeffizient des elektrischen Widerstands $\left(T K_{R}\right)$ von verschiedensten Ni-DLCSchichten. Der TK $K_{R}$ ist mit Hilfe des Nickelgehalts auf Werte nahe Null einstellbar. 


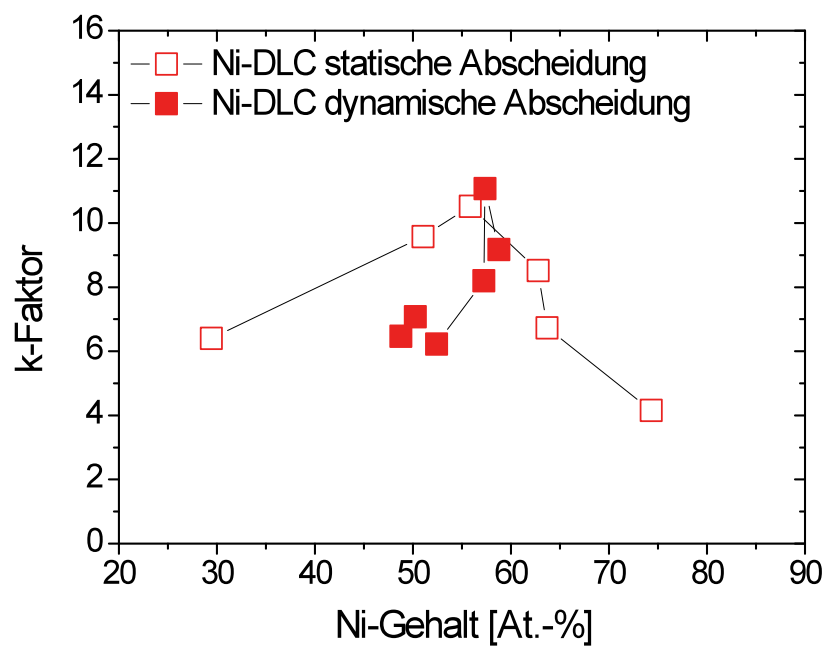

Abbildung 8: Dehnungsempfindlichkeit (k-Faktor) von Ni-DLC mit nicht optimierten Prozessparametern auf statischen und rotierenden (dynamischen) Substraten.

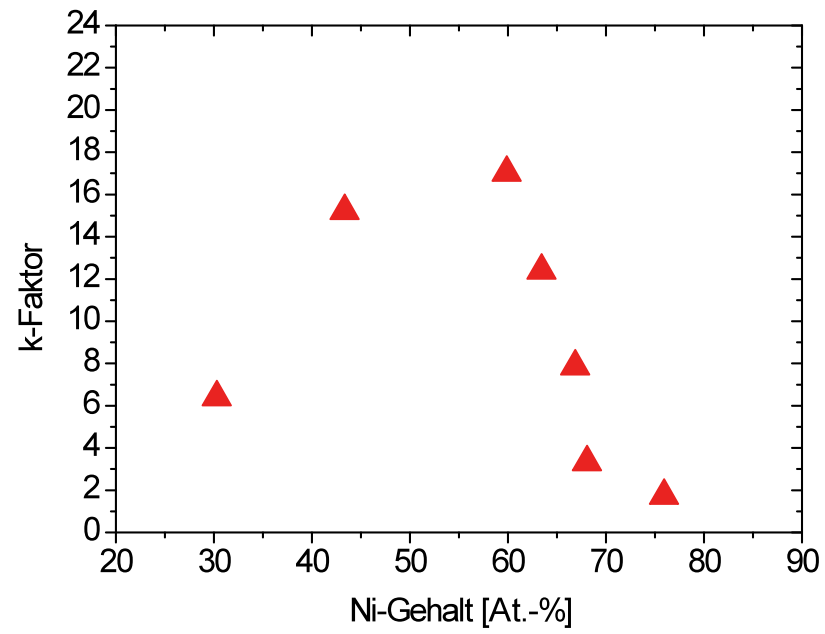

Abbildung 9: Dehnungsempfindlichkeit von optimiertenNi-DLC-Schichten in Abhängigkeit vom Ni-Gehalt.

Abbildung 10 zeigt die Widerstandsdrift verschiedener Ni-Cr-Legierungen über 250 Stunden bei 100 bzw. $150{ }^{\circ} \mathrm{C}$ vor und nach dem etwa 10 -stündigen Tempern bei gut $300{ }^{\circ} \mathrm{C}$. Deutlich zu erkennen ist, dass die im ungetemperten Zustand deutlich instabilere NiCr40/60-Legierung nach dem Temperschritt eine ebenso geringe Widerstandsdrift (ca. 0,2-0,3 ppm/h) zeigt wie dieNiCr80/20-Legierung. Erste Ergebnisse mit Ni-DLC zeigen eine mit $3,9 \mathrm{ppm} / \mathrm{h}$ ebenfalls geringe Drift bei $100^{\circ} \mathrm{C}$. 


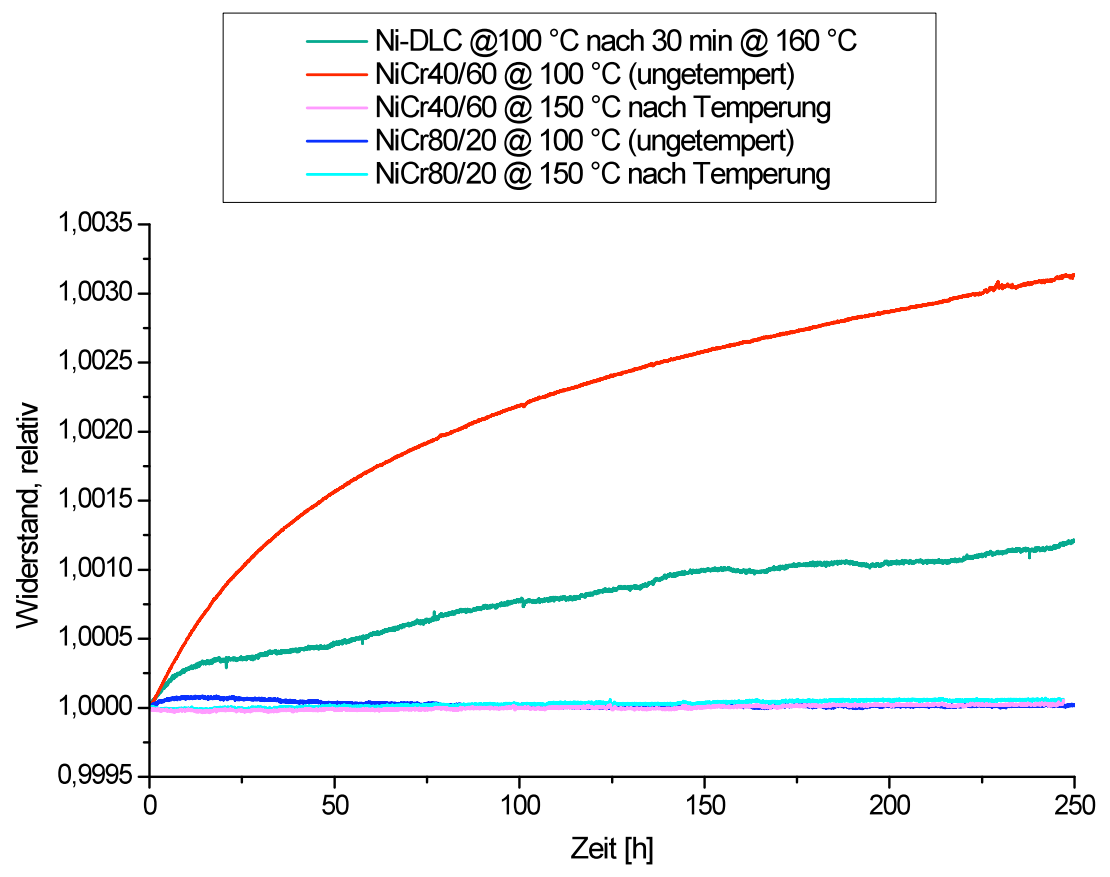

Abbildung 10: Widerstandsdrift unter Umgebungsbedingungen von 100 und $150{ }^{\circ} \mathrm{C}$ von Ni-DLC- und verschiedenen NiCr-Schichten auf Testsubstraten.

\section{Strukturierung}

Während üblicherweise metallische Sensorschichten mittels Photolithographie strukturiert und nasschemisch geätzt werden, muss für Ni-DLC ein alternativer Prozess entwickelt werden. Da DLC chemisch inert ist, kann z. B. auf Plasmaätzen zurückgegriffen werden. Die Strukturierung erfolgt mit etablierter Photolithographie, wobei die Lackdicken an die Selektivität des Prozesses angepasst werden müssen. Für die Strukturierung werden anschließend verschiedene Ätzgasmischungen (primär $\mathrm{Ar} / \mathrm{O}_{2}-$ Gasgemische) betrachtet. Alternativ kann auch eine zusätzliche metallische Maskierung erfolgen, die nach dem Plasmaätzen nasschemisch entfernt wird. Aber auch die Strukturierung mit dem Pikosekunden-Laser, die sich bereits bei der Strukturierung von NiCr-DMS auf $\mathrm{Al}_{2} \mathrm{O}_{3}$-Isolationsschicht und dreidimensionalem metallischen Substraten bewährt hat [Sut11], ist eine mögliche Option zur Strukturierung von Dehnungsmessstreifen auf der Basis vonNi-DLC.

\section{Zusammenfassung}

Kommerzielle Drucksensoren auf Dünnschichtbasis werden in einer Vielzahl von Produkten zur Messung von statischen oder dynamischen Drücken eingesetzt. Die moderne Beschichtungstechnik kann zur Erhöhung der Messgenauigkeit beitragen, indem die Sensorschicht direkt auf die Oberfläche des Bauteils aufgebracht wird. Anstelle der Klebeschicht und der ca. $50 \mu \mathrm{m}$ starken Trägerfolie eines herkömmlichen Folien-DMS wird eine nur $5 \mu \mathrm{m}$ dünne Isolationskeramikschicht benötigt, um die gewünschte Durchschlagsspannung von mehr als $500 \mathrm{~V}$ zwischen Sensor und Bauteil zu erreichen. Voraussetzung ist die möglichst weitgehende Vermeidung von Partikeln auf der zu beschichtenden Substratoberfläche, welches durch geeignete Konstruktion der Beschichtungsanlage zuverlässig realisiert werden kann. Weiter gesteigert wird die Messgenauigkeit der Sensoren auf DMS-Basis durch eine erhöhte Dehnungsempfindlichkeit der Sensorschicht. Hier bietet insbesondere Ni-DLC mit einer 5- bis 10-fach erhöhten Dehnungsempfindlichkeit bei gleichzeitiger Temperaturkompensation und niedrigerWiderstandsdrift ein hohes Potenzial. Die aus der Mikrotechnologie bekannten Strukturierungsmethoden wie Lithographie (inkl. Lift-off-Technik) und Laserstrukturierung mit dem Pikosekundenlaser komplettieren die Prozesskette zur Herstellung von hochempfindlichen (Druck)Sensoren in Dünnschichttechnik. 


\section{Literatur}

[Aie11] Aiempanakit, M.;Kubart, T.;Larsson, P.; Sarakinos, K.; Jensen, J.; Helmersson, U.: Hysteresis and process stability in reactive high power impulse magnetron sputtering of metal oxides. In: Thin Solid Films, Vol. 519, 22 (2011) S. 7779-7784.

[Ban09] Bandorf, R.; Heckmann, U.; Lübke, M.; Schnabel, S.; Bräuer, G.: Development of Me-DLC Films for Strain Gauge Application. In: SVC Proc., 52nd Annual Technical Conference (2009) S. 26-30.

[Bar02] Bartzsch, H.;Frach, P.;Goedicke, K.; Böcher, B.;Gottfried, Chr.: Ensuring long-term stability of process and film parameters during target lifetime in reactive magnetron sputtering. In: Surface and Coatings Technology, Vol. 150, 1 (2002) S. 88-94.

[Bie06] Biehl, S.; Lüthje, H.; Bandorf, R.; Sick,J.-H.: Multifunctional thin film sensors based on amorphous diamond-like carbon for use in tribological applications. In: Thin Solid Films, Vol. 515 (2006) S. 1171-1175.

[Cha80] Chapman, B.: Glow Discharge Processes: Sputtering and Plasma Etching. New York: John Wiley \& Sons, 1980.

[Due11] Duesing, J.F.; Suttmann, O.;Bandorf, R.;Gerdes,H.:Oberflächen-Dünnschichtsensorik erschließt neue Anwendungen. In: Laser Magazin, Ausgabe 3 (2011) S. 11-12.

[Fru05] Joachim Frühauf: Werkstoffe der Mikrotechnik, München: Carl Hanser Verlag, 2005.

[Glo11] GlobischS. (Hrsg.): Lehrbuch Mikrotechnologie, München: Carl Hanser Verlag, 2011.

[Kel96] Kelly, P.J.; Abu-Zeid,O.A.;Arnell, R.D.; Tong, J.:The deposition of aluminium oxide coatings by reactive unbalanced magnetron sputtering. In: Surface and Coatings Technology, Vol. 86-87, 1 (1996) S. 28-32.

[Kop09] Koppert, R.; Göttel, D.; Freitag-Weber, O.;Schultes, G.: Nickel containing diamond like carbon thin films. In: Solid State SciencesVol. 11, 10 (2009) S. 1797-1800.

[Kop10] Koppert, R.;Göttel, D.;Schultes, G.;Werner, U.: NanoNi@C: Hochempfindliche Funktionsschicht für Druck- und Kraftsensoren. In: Technisches Messen 77, 12 (2010) S. 631-637.

[Lue99] Lüthje, H.; Brand,J.: Sensor zur Zustandsbestimmung von Kenngrößen an mechanischen Komponenten, DE 19954164 A1, 1999.

[Mar91] Maruyama, T; Nakai, T.: Aluminum oxide thin films prepared by chemical vapor deposition from aluminum 2-ethylhexanoate. In: Applied Physics Letters, Vol. 58, 19 (1991) S. 2079-2080.

[Mat10] Mattox, D. M. (Hrsg.): Handbook of Physical Vapor Deposition (PVD) Processing, $2^{\text {nd }}$ Edition, Elsevier, 2010.

[Par63] Parker, R. L.; Krinsky, A.: Electrical Resistance-Strain Characteristics of Thin Evaporated Metal Films. In: Journal of Applied Physics, Vol.34, 9 (1963) S.2700-2708.

[Pet11] Petersen, M.;Heckmann, U.; Bandorf, R.; Gwozdz, V.;Schnabel, S.;Bräuer, G.;Klages,C.-P.: Me-DLC films as material for highly sensitive temperature compensated strain gauges. In: Diamond and Related Materials,Vol. 20, 5-6 (2011) S. 814-818.

[Pet12] Petersen, M.; Bandorf, R.; Bräuer, G.; Klages C.-P.: Diamond-like carbon films as piezoresistors in highly sensitive force sensors. In: Diamond and Related Materials, eingereicht(vermutl. 2012).

[Sch97] Schneider, J.M.;Sproul, W.D.; Chia, R.W.J.;Wong, M.-S.; Matthews, A.: Very-high-rate reactive sputtering of alumina hard coatings. In: Surface and Coatings Technology, Vol. 96, 2-3 (1997) S. 262-266.

[Smi54] Smith, C.S.: Piezoresistanceeffekt in germanium and silicon. In: Physical Review, Vol. 94, 1 (1954) S. 42-49.

[Sut11] Suttmann, O.; Duesing, J. F.; Klug, U.; Kling, R.: Patterning of $\mathrm{NiCr}(80 / 20)$ on $\mathrm{Al}_{2} \mathrm{O}_{3}$ Using Picosecond Laser Pulses: In:Journal of Laser Micro/Nanoengineering, Vol. 6, 1 (2011) S. 31-36.

[Tib06] Tibrewala, A.; Peiner, E.; Bandorf, R.; Biehl, S.; Lüthje, H.: Transport and optical properties of amorphous carbon and hydrogenated amorphous carbon films. In:Applied Surface Science, Vol. 252, 15 (2006) S. 5387-5390.

[Ver11] Vergöhl, M.; Bandorf, R.; Bräuer, G.; Bruns, S., Werner, O.: Reactive HiPIMS of Oxides for Optical Coatings.In: SVC Proc., 54 ${ }^{\text {th }}$ Annual Technical Conference (2011) S.121-130.

[Wal08] Wallin, E.; Helmersson, U.: Hysteresis-free reactive high power impulse magnetron sputtering. In: Thin Solid Films, Vol. 516, 18 (2008) S. 6398-6401. 